\title{
DES ÉPHÉMÉRIDES ASTRONOMIQUES ANNUELLES EN PRÉLIMINAIRE À L'ANNUAIRE DU BUREAU DES LONGITUDES
}

\author{
SUZANNE DÉBARBAT \\ Observatoire de Paris - DANOF/URA 1125 \\ 61 avenue de l'Observatoire, F-75014 Paris
}

La Convention nationale, après avoir entendu le rapport de ses Comités de marine, des finances et d'instruction publique, décrète:

ARTICLE PREMIER

Il sera formé un Bureau des longitudes.

$$
\text { V. }
$$

Le Bureau des longitudes est chargé de rédiger la connoissance des temps, qui sera imprimée aux frais de la République, de manière que l'on puisse toujours avoir les éditions de plusieurs années à l'avance; il perfectionnera les tables astronomiques, et les méthodes des longitudes, et s'occupera de la publication des observations astronomiques et météorologiques. (Extrait du Rapport sur l'établissement du Bureau des longitudes par Grégoire, Séance du 7 Messidor, l'an 3 de la République une et indivisible; Suivi du décret de la Convention nationale, et Imprimé par son ordre. Le document, de seize pages se termine par la mention: Adopté; il est dit: A Paris, De l'Imprimerie nationale, Messidor l'An III)

Si l'on en croit Lalande (1732-1807) et sa Bibliographie astronomique (1803), la plus ancienne des éphémérides conservées date de 1150 (Tabulae astronomicae et ephemerides, R. Salomon Iarchi, Iarchus). Au 15e siècle, tables et almanachs, calendriers et éphémérides se partagent la vedette et, à propos de Regiomontanus (Johann Muller, 1436-1476), Lalande note que Ces éphémérides sont les premières qui aient été publiées et, pour ainsi dire, les premières qui aient été faites. Elles couvrent les années 1475 à 1506 . C'est d'ailleurs au siècle suivant que vont être principalement publiées des éphémérides pour plusieurs années successives: 1536-1550 (15 ans); $1552-1562$ (11); $1554-1568$ (15); $1554-1570$ (17); 1557-1575 (19); 15581577 (20); 1564-1584 (21); 1576-1600 (25); $1577-1590$ (14); 1581- 1615 (35); 1584-1607 (24); 1589-1600 (12); 1590-1610 (21); 1595-1630 (36). La durée couverte est variable, mais toujours longue; le "clou" est une publication (Paris, 1571) in-quarto Ephemerides, ou almanach du jour et de la nuit, pour cent ans, et celui de la lune durant le temps de 19 ans; composé et 
revu par J. Gosselin de Vize. Il est vrai que les voyages à la mer, nécessitant des connaissances astronomiques, duraient de nombreuses années... Le 17e siècle, qui permet à Képler (1571-1630) l'exploitation des observations de Tycho Brahe (1546-1601), voit se publier à la fois des éphémérides de longue durée: 1607-1618 (12); 1609-1617 (9); 1621-1640 (20); 1629-1640 (12); 16371700 (64); 1641-1660 (20); 1659-1671 (13); 1661-1675 (15); 1672-1681 (10); $1666-1680(15) ; 1675-1684(10)$, et des éphémérides de durée plus réduite: 1633-1636 (4); 1636-1640 (5); 1638-1642 (5); 1682-1684 (3). Cependant, vont paraître aussi des éphémérides annuelles, pour 1615 par exemple, pour 1617 , pour $1650,1666,1683,1685, \ldots$

Dans le même temps se crée la Connoissance des tems ou calendrier et éphémérides... pour l'année 1679, calculées sur Paris... car, indique Lalande, Les éphémérides de Hecker couvraient les années 1666 à 1680. Il est bien connu qu'avec cette Connoissance des tems de 1679 commençait une série d'éphémérides annuelles dont l'année 1995 marque le $317 \mathrm{e}$ volume. Il est bien connu, aussi, que l'Adresse $A u$ Roy de ce premier volume précise qu'il a été établi après l'avoir épuré de toutes les choses ridicules dont ces sortes d'Ouvrages ont esté remplis jusqu'à présent. Éphémérides remplies de choses ridicules et aussi éphémérides ridiculisant leurs auteurs cohabitaient puisqu'à propos d'un in-octavo paru, en allemand en 1607, Lalande écrivait qu'il s'agissait de La grande mère de tous les almanachs, ou almanach universel, qui ne contient que des prédictions ridicules des choses assurées; fait pour se moquer des astrologues. Le nom de calendrier, d'éphéméride, de table, d'almanach est souvent usité de manière indifférente pour des publications contenant des indications astronomiques. Le terme d'almanach sera d'ailleurs celui retenu en Grande-Bretagne quand paraîtra, créé en 1767, le Nautical Almanac.

Malgré les remarques de Lalande, la confusion des contenus continuera au 18e siècle, mêlant d'ailleurs, pour le public, astronomie, astrologie, météorologie ainsi qu'en témoigne un Almanach astrologique pour l'année 1769 qui marque exactement le tems de chaque jour, excepté les tems d'orage (Strasbourg, 1769). La CLEF qu'il contient donne sous forme de pictogrammes les conditions météorologiques et l'on constate que celles-ci figurent, effectivement, à chacun des jours de l'année... Un Calendrier royal pour l'année 1790 est beaucoup plus prudent puisqu'il ignore les prédictions météorologiques tout comme d'autres calendriers lesquels constituent, au $18 \mathrm{e}$ siècle, les éphémérides populaires, se limitant à l'énoncé des jours et des saints de l'année (Paris, 1741) même lorsque, plus tard, ils sont présentés à la Nation, la Loi et le Roi, à Paris en 1792.

Quant aux scientifiques du 18e siècle, peu soucieux de laisser prise aux croyances astrologiques, ils publient des volumes d'éphémérides strictement 


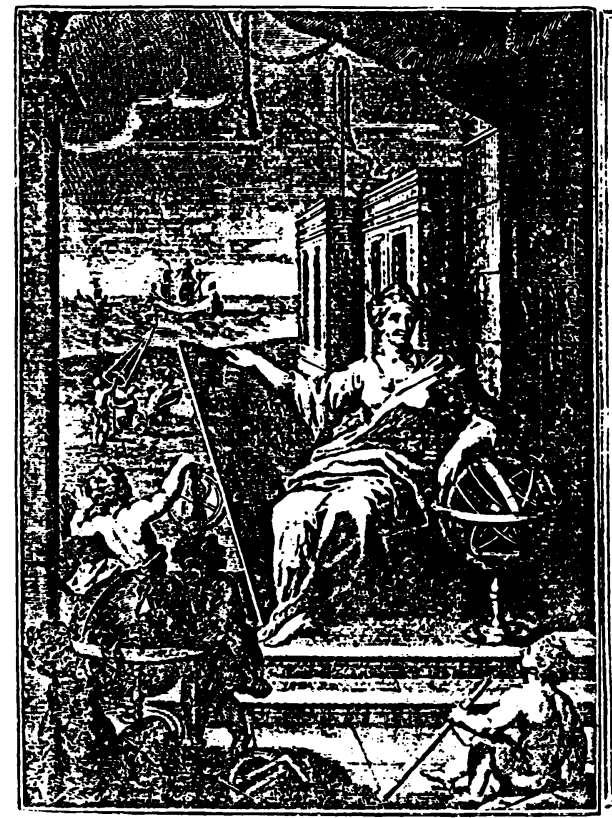

\section{EPHEMERIDES \\ DES MOUVEMENS \\ C E L E S T E S.}

POUR LES ANNE'ES 17IS, JUSQU'EN 1725.

OS l'on inuve les mouvemens diurnes des Plinetes en I.ongirude: leurs Latrudes, Alpects \& Mediations; celles des

Etoiles, leur levir , coucher, apparitions \& occultations ; les Inmerfions \& Emerfions du prem.er Sat ilie die Jupatier pour les inèmes années.

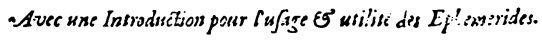
pOLR LE MiEzIDIEN dE LA VILLE De TARIS.

Par le Sicur Despla ces.

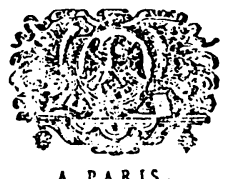

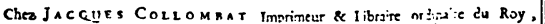

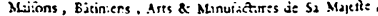
nox $S$ Jucques, au Pelizi

ATEC APPEOBATION ET PRIILECE DIV KOOY

Figure 1. Frontispice et page de titre des éphémérides de Desplaces pour les années 1715 à 1725 . (Collection particulière)

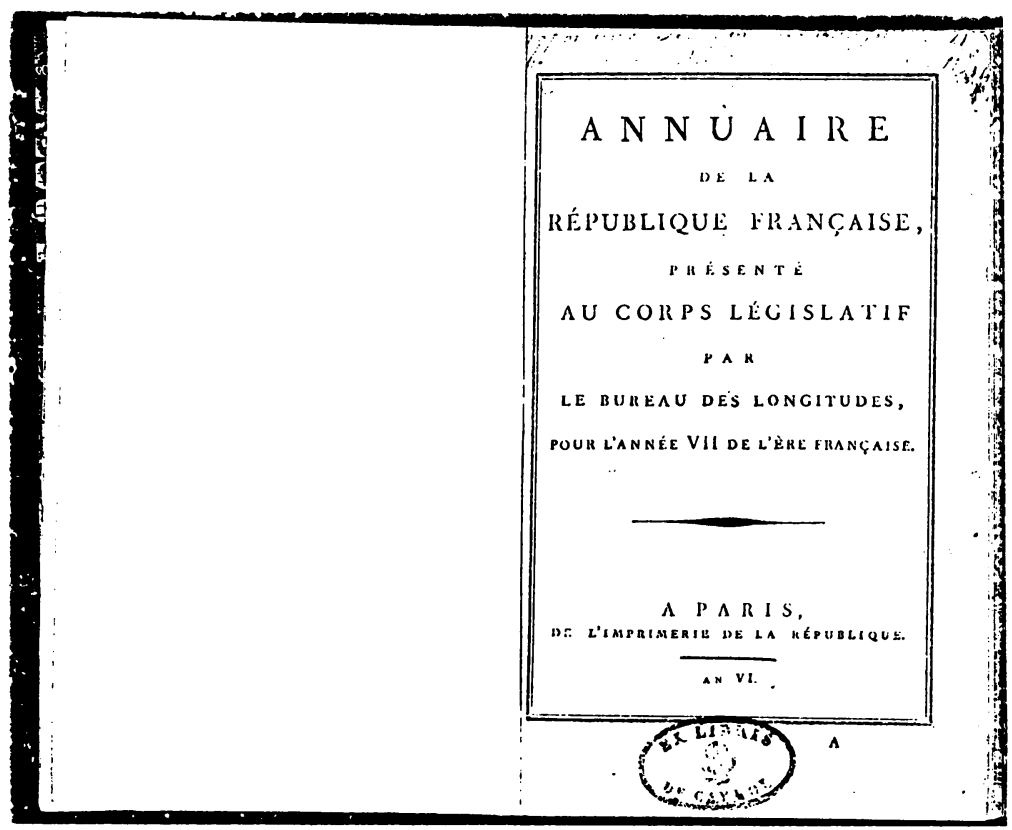

Figure 2. Page de titre de l'Annuaire pour l'année VII. (Collection du Bureau des longitudes 


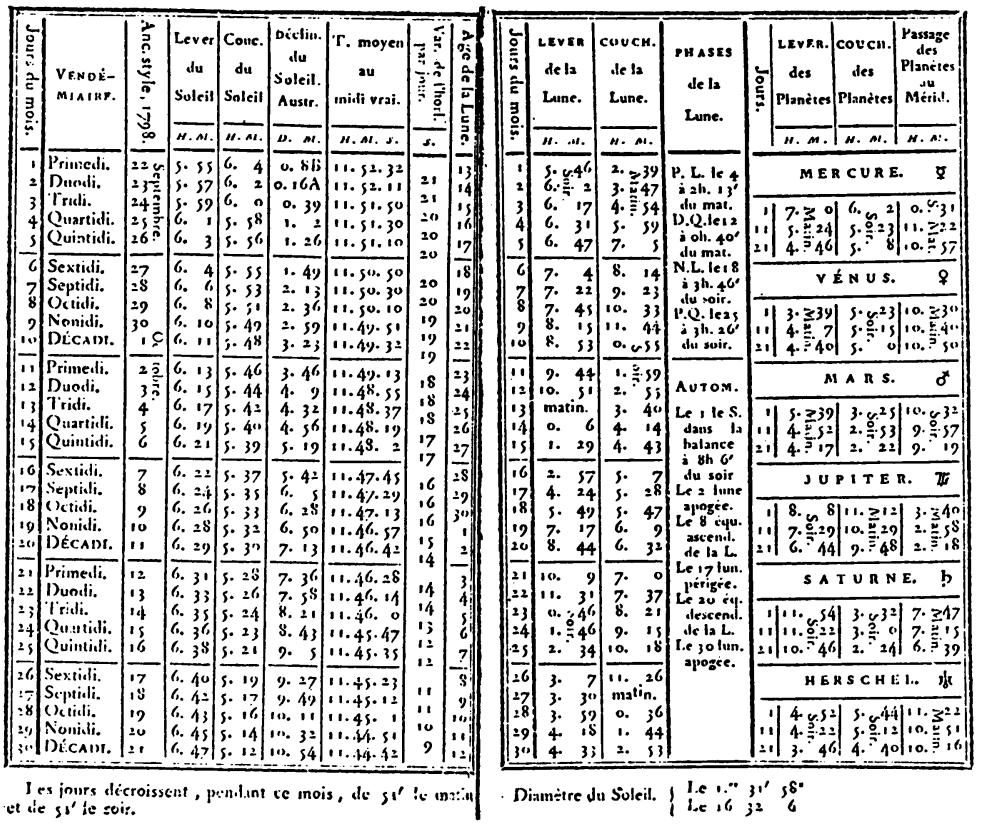

Figure 3. Pages 8 et 9 de l'Annuaire pour l'année VII. (Collection du Bureau des longitudes

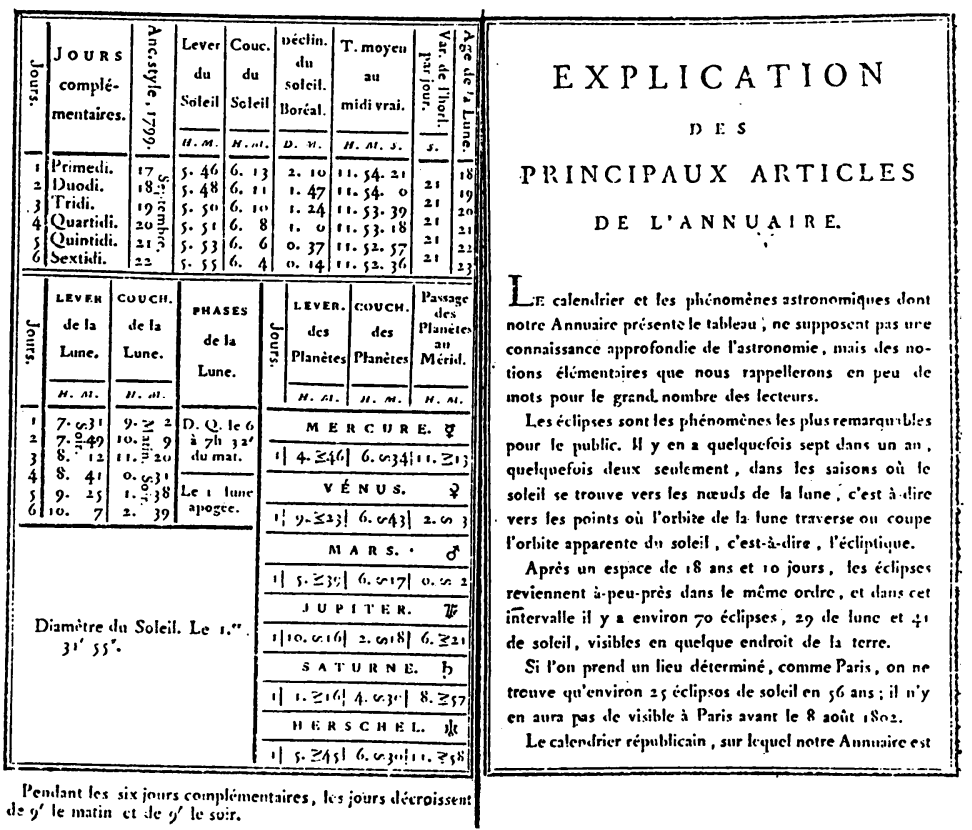

Figure 4. Pages 32 et 33 de l'Annuaire pour l'année VII. (Collection du Bureau des longitudes 
consacrés aux éléments astronomiques tels que levers et couchers du Soleil et de la Lune, positions des planètes, etc. En France, la Connaissance des temps poursuit sa route annuelle depuis son premier volume de 64 pages. Lalande précise, à son propos: Lefebvre y mit son nom en 1685, Lieutaud en fut chargé en 1702, Godin en 1730, Maraldi en 1734 (Godin va partir pour l'expédition du Pérou). Puis il indique: J'ai commencé en 1760, Jeaurat en 1776, Méchain en 1788; J'ai repris la rédaction de l'an 4 (1795) et des années suivantes. Ainsi Lalande se trouve en charge de cette éphéméride quand le Bureau des longitudes est créé par la Loi du 7 Messidor an III (25 juin 1795).

Trois mois plus tard, le $4 \mathrm{e}$ jour complémentaire An III (20 septembre 1795), est édicté le Règlement du Bureau des longitudes dont l'Article 9 est ainsi rédigé: Le Bureau présentera chaque année au Corps législatif un Annuaire propre à régler ceux de toute la République. Tout au long $\mathrm{du} 18 \mathrm{e}$ siècle dans différentes villes d'Europe, telles Berlin, Bologne, Londres, Venise, Leipzig, Stockholm, Edinburgh, Florence, Vienne, Hamburg, Amsterdam, Milan, Lisbonne, Rome,... nombre d'ouvrages d'éphémérides seront publiés. En France, à Rouen, dès 1701 - en in-quarto - paraissent des éphémérides annuelles que commente Lalande: (Jean de Baulieu, ou Desforges) Le même auteur en donna, en 1703, la suite jusqu'en 1715 que commencèrent les éphémérides de Desplaces, continuées en 1745 par Lacaille, et par moi depuis 1775 jusqu'en 1800. La figure 1 donne, du volume de la période 1715-1725 (en fait couvrant les années 1715-1724), le frontispice et la page de titre détaillant les éléments figurant chaque année. En fait Desforges (décédé en 1714) avait repris le nom de Beaulieu, mathématicien de Paris qui avait calculé des éphémérides avant lui.

Ces éphémérides ont couvert les périodes successives: 1715-1725; 1725 $1735 ; 1735-1745 ; 1745-1755 ; 1755-1765 ; 1765-1775 ; 1775-1784 ; 1784-1793$; 1793-1800. Chaque volume comprend essentiellement, pour chaque année, 26 pages dont deux pour chaque mois: à gauche Mouvemens diurnes des planètes (dont le Soleil et la Lune), à droite Aspects des planètes incluant dans les FESTES les saints du jour avec, en bas de page, les immersions et les émersions du ler satellite de Jupiter. Le dernier volume de la publication commencée par Desplaces (1659-1736), poursuivie par Lacaille (1713-1762) puis Lalande, est donc paru quand - en 1795 - ce dernier prend en charge la Connaissance des temps, au titre du Bureau des longitudes, et qu'est décidée, le 20 septembre 1795, la création d'un Annuaire. Dans l'introduction de ce volume (1793-1800), Lalande précise: La connoissance des Tems que M. Méchain vient de publier pour 1792, contient sur les Mouvemens célestes de plus grands détails, ainsi que les Ephémérides de Londres, de Berlin, de Milan, de Vienne en Autriche; mais les nôtres offrent, plusieurs années d'avance, tout ce qui suffit au Public et même 
à des Astronomes qui, allant dans des Pays éloignés, ne pourroient pas se procurer les ouvrages plus étendus. Dans ce volume, toujours à deux pages par mois, figurent les immersions et les émersions des quatre satellites de Jupiter.

Le premier volume de l'Annuaire de la République française comme il s'appelle alors, ne paraîtra qu'en juillet 1796, l'an IV de la République française. Il sera établi pour l'année $V$ de l'ère française (1797 ancien style). La figure 2 montre la page de titre de l'Annuaire pour l'année VII. L'Avertissement précise qu'il est extrait de la Connaissance des tems et qu'il doit parvenir facilement et en nombre suffisant dans toutes les parties de la France. Les éléments sont évidemment donnés dans le Calendrier républicain (figure 3 ) incluant, néanmoins (à partir, comme il se doit, du début de l'année républicaine, le 22 septembre 1798), les dates ancien style. Les éphémérides des levers et couchers de la Lune et du Soleil (figures 3 et 4), qui constituent l'essentiel du volume, se terminent par les 6 jours complémentaires de l'an VII (17 au 22 septembre 1799).

De dimensions fort modestes par rapport aux volumes des éphémérides précédemment établies, par Desplaces et les autres, le nouvel Annuaire propre à régler ceux de toute la République se révélait plus maniable pour atteindre les populations (sachant lire) et sans doute aussi moins coûteux pour le budget de l'époque; son prix, pour plusieurs années, sera de 60 centimes. C'est cet Annuaire qui, en 1995, se présente dans un format à peine plus petit que le volume qui, en 1800 , terminait la série des Ephémérides des mouvements célestes initialisée au début du 18e siècle, mais qui compte - pour chaque année - environ 300 pages. Quant à l'Almanach des postes, il suffit - d'après les données du Bureau des longitudes - à fournir, au public, une très large part de ce que contenait l'Annuaire de l'An VII, puisqu'il comprend les levers et couchers de la Lune et du Soleil. Pour ceux, d'ailleurs, auxquels ces éléments ne suffiraient pas, ils peuvent se rapporter à l'Annuaire du Bureau des longitudes de l'année en cours, sous-titré Ephémérides astronomiques ou au Minitel, puisqu'il existe depuis plusieurs années le $3616 \mathrm{BDL}$.

\section{Références}

Annuaires du Bureau des longitudes pour les années V, VI, VII.

Connaissances des temps des années 1679 , an IV, an V, an VI.

Ephémérides des mouvemens célestes de Desplaces, 1715 à 1800.

Rapport sur l'établissement du Bureau des longitudes par Grégoire, Séance du 7 Messidor, l'an 3 (24 juin 1795) de la République une et indivisible.

Règlement du Bureau des longitudes du $4 \mathrm{e}$ jour complémentaire an III (20 septembre 1795).

Lévy, J.: 1976, "La création de la Connaissance des temps" Vistas in Astronomy 20, 75-77. 